Bull. Mater. Sci., Vol. 14, No. 4, August 1991, pp. 1029-1032. (C) Printed in India.

\title{
Superconducting gap function and upper critical field in the presence of inhomogeneous magnetic order
}

\author{
P C PADHI ${ }^{\dagger}$, S PANIGRAHI ${ }^{\dagger \dagger}, \mathrm{S}$ DAS and N C DAS* \\ Department of Physics, Berhampur University, Berhampur 760007, India. \\ Present Address: ${ }^{\dagger}$ Department of Physics, Aska Science College, Aska 761 111, India. \\ ${ }^{+\dagger}$ Department of Physics, Indira Gandhi Institute of Technology, Sarang, \\ Talcher 759 100, India.
}

\begin{abstract}
Rare earth ternary superconductors are known to exhibit oscillatory magnetic orders below their superconducting transition temperatures. The study of behaviour of superconducting electrons in an inhomogeneous magnetic field is therefore important for such systems. We report here the results of our theoretical study of superconducting gap function $\Delta(T)$ and upper critical field $H_{\mathrm{c} 2}(T)$. The results are applied to analyse and explain the variation of $\Delta(T)$ and $H_{c 2}(T)$ in case of $\mathrm{NdRh}_{4} \mathrm{~B}_{4}$.
\end{abstract}

Keywords Ternary superconductor; oscillatory magnetic order; gap function; upper critical field.

\section{Introduction}

The variations of superconducting gap function $\Delta(T)$ and the upper critical field $H_{c 2}(T)$ with temperature form two important aspects of study of a superconductor. In rare earth ternary superconductors oscillatory magnetic orders are known to exist below their superconducting transition temperatures $\left(T_{c}\right)$. In such cases the gap function $\Delta(T)$ and upper critical field $H_{c 2}(T)$ are profoundly modulated by the occurrence of inhomogeneous magnetic field inside the material. The rhodium boride compounds $\mathrm{R} \mathrm{Rh}_{4} \mathrm{~B}_{4}(\mathrm{R}$ denoting rare earth) provide a wealth of experimental results on the interplay of superconducting and magnetic phenomena. Compounds of class of $\mathrm{R} \mathrm{Rh}_{4} \mathrm{~B}_{4}$ with $\mathrm{R}=\mathrm{Sm}, \mathrm{Nd}$, Tm, display co-existence of superconductivity and antiferromagnetism while those of other class with $R=G d, T h, D y, H o$ and $\mathrm{Er}$ undergo ferromagnetic ordering at low temperature leading to disappearance of superconductivity. Reviews of experimental results (Maple et al 1982) particularly on magnetic ordering are available (Misra and Postma 1984) from earlier works. Here $\mathrm{NdRh}_{4} \mathrm{~B}_{4}$ attracts special attention with its two magnetic order transitions at critical temperature $T_{N 1}$ and $T_{N 2}$ below its superconducting transition temperature $T_{c}$. The wavelength and direction of the periodic magnetic order developed at $T_{N 1}$ changes as the system reaches $T_{N 2}$. The curve of $H_{c 2}(T)$ versus $T$ shows one $U$-shaped depression between $T_{N 1}$ and $T_{N 2}$. In the present work we apply our theory to study the variation of $\Delta(T)$ and $H_{c 2}(T)$ of $\mathrm{NdRh}_{4} \mathrm{~B}_{4}$ with temperature and try to analyse and explain the anomalous variation of $H_{\mathrm{c} 2}(T)$.

*For correspondence 


\section{Theory}

A model Hamiltonian (Panigrahi and Das 1989) is considered to describe the system in the presence of an inhomogeneous magnetic order represented by the magnetization density $\mathbf{M}(\mathbf{r})=\mathbf{M}_{\mathbf{Q}} \cos \mathbf{Q} \cdot \mathbf{r}$ in the system. This includes the reduced BCS interaction, the exchange interaction with localized spins on the lattice and the magnetic dipole interaction between the magnetic induction field and the local spin magnetization. This magnetic dipole interaction couples superconducting electrons to local spins via magnetic induction field and gives rise to electromagnetic interaction between local spins and superconducting electrons. We use thermodynamic Green's functions $G$ and $F^{\dagger}$ and set up an equation (Das 1984) for the gap function. We solve this equation to obtain expressions for the gap function $\Delta(T)$ and the upper critical field $H_{c 2}(T)$ in appropriate limits. The expressions for $\Delta(T)$ and $H_{c 2}(T)$ are given by

$$
\begin{gathered}
\Delta^{2}=\frac{N^{2}(\mu)}{B(T) N(0)}\left(\ln \frac{T}{T_{c}}+\frac{\pi \hbar \delta}{4}\left(;-\beta_{c}\right)+\left(\Phi_{1}-\Phi_{1}^{c}\right)\right)+\frac{4 \pi^{2} N(\mu)}{3 \rho_{0}^{2} \xi_{0}^{2} B(T)}\left(\Phi_{2}-\Phi_{2}^{c}\right) \\
\frac{H_{c 2}(T)}{H_{c 2}(0)}=\frac{1}{1+4 \pi \chi} \frac{N(\mu)}{N(0)}\left[\frac{6 \xi_{0}^{2}}{\alpha(T)} \ln \frac{T_{c}}{T}-\frac{3}{2} \frac{\pi \xi_{0}^{2} \hbar \delta}{\alpha(T)}\left(\beta-\beta_{c}\right)-\frac{6 \xi_{0}^{2}}{\alpha(T)}\left(\Phi_{1}-\Phi_{1}^{c}\right)\right. \\
\left.-\frac{8 \pi^{2}}{\rho_{0}^{2} \alpha(T)}\left(\Phi_{2}-\Phi_{2}^{c}\right)\right]\left[1+\frac{4 \pi n_{y}}{\rho_{0}^{2}} \frac{M_{Q}}{H_{c 2}^{0}(0)}\left\{1+\rho n_{0}(\rho)\right\}\right]^{-1}
\end{gathered}
$$

where $\Phi_{1}=\frac{a}{b}\left(1+\frac{\mu}{b}\right) \operatorname{Re}[\Psi(z)-\Psi(x)]$

$$
\begin{aligned}
\Phi_{2} & =\alpha(T)\left[M_{Q}(T) / H_{c 2}^{0}(0)\right]^{2}\left[1-j_{0}(2 \rho)\right], \\
a & =A_{Q} / 2 \varepsilon_{Q}, \quad b=\mu+a \\
A_{Q} & \simeq\left(J^{2} M_{Q}^{2} / 16\right), \quad \varepsilon_{Q}=\hbar^{2} Q^{2} / 2 m . \\
x & =\frac{1}{2}+\beta \hbar \delta / 2 \pi, \quad z=x+i \beta b / 2 \pi .
\end{aligned}
$$

In the above $M_{Q}$ is the staggered magnetization of wave vector $Q, \xi_{0}$ is the superconducting coherence length at $T=0$, the parameter $\rho_{0}=Q \xi_{0}, \beta=1 / K_{B} T, \Phi_{1}^{c}, \Phi_{2}^{c}$, $\beta_{c}$ refer to the values at $T=T_{c} \cdot \mu$ is the chemical potential, $N(\mu)$ and $N(0)$ are densities of states at fermi level in the presence and absence of magnetic ions, respectively. $\chi$ is the average uniform magnetic susceptibility, $H_{c 2}^{0}(0)$ is the upper critical field of spin-free system at $T=0$. The other symbols have been defined in earlier text (Panigrahi and Das 1989).

\section{Numerical results}

We have used equation (1) to study the variation of $\Delta(T)$ with temperature. The antiferromagnetic superconductor $\mathrm{NdRh}_{4} \mathrm{~B}_{4}$ has $T_{c}=5 \cdot 3 \mathrm{~K}$. A magnetic ordering with $\lambda=46.5 \AA$ develops in the $(100)$ direction at $T_{N 1}=1.31 \mathrm{~K}$ which then changes to an order with $\lambda=45.2 \AA$ in (110) direction at $T_{N 2}=0.89 \mathrm{~K}$. We have calculated the values of $\Delta(T)$ between $5.3 \mathrm{~K}$ and $0.9 \mathrm{~K}$ considering the first magnetic order along (100) 
direction with $T_{N 1}=1.31 \mathrm{~K}$. Below $0.9 \mathrm{~K}$ we have taken the magnetic order in (110) direction with $T_{N 2}=0.89 \mathrm{~K}$. Temperature variation of staggered magnetization $M_{Q}(T)$ is calculated from $M_{Q}(T)=M_{Q}(0)\left(T_{N}-T\right)^{0.33}$. The variation of $\Delta(T)$ with temperature is presented in figure 1 for a fixed value of $M_{Q}(0) / H_{c 2}^{0}(0)=0.9$. Figure 2 shows the variation of $\Delta(T)$ with staggered magnetization $M_{Q}(T)$ at a fixed temperature. The parameters used for the calculation are shown in table 1 .

We have used equation (2) for calculating $H_{c 2}(T)$ of $\mathrm{NdRh}_{4} \mathrm{~B}_{4}$ along the easy axis of magnetization. We have taken into account the effects of two magnetic transitions below its $T_{c}=5.3 \mathrm{~K}$. We have calculated $H_{c 2}(T)$ in the range $5.3 \mathrm{~K}$ to $0.9 \mathrm{~K}$ considering the periodic magnetic order of $\lambda=46.5 \AA$ along $(100)$ direction with $T_{N 1}=1.31 \mathrm{~K}$. For $H_{c 2}(T)$ below $0.9 \mathrm{~K}$, we have considered the magnetic order $\lambda=45.2 \AA$ along (110) direction and $T_{N 2}=0.89 \mathrm{~K}$. The choice of appropriate value of $n_{y}$ (Panigrahi and Das 1989) (i.e. $n_{y}=1$ for $M_{Q}$ in (100) direction and $n_{y}=1 / \sqrt{2}$ for $M_{Q}$ in (110) direction) has been made for calculation. The other parameters used are the same as in the case

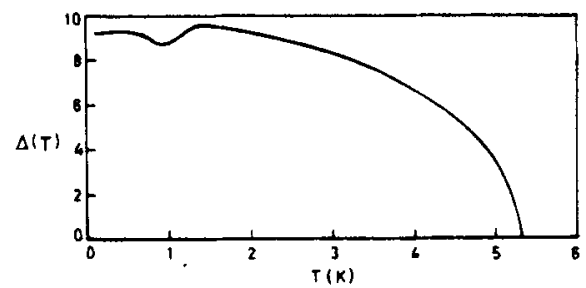

Figure 1. Variation of superconducting gap function $\Delta(T)$ with temperature $T(K)$ for $M_{Q}(0) / H_{c 2}(0)=0.9 . \Delta(T)$ is in units of $10^{-4} \mathrm{eV}$.

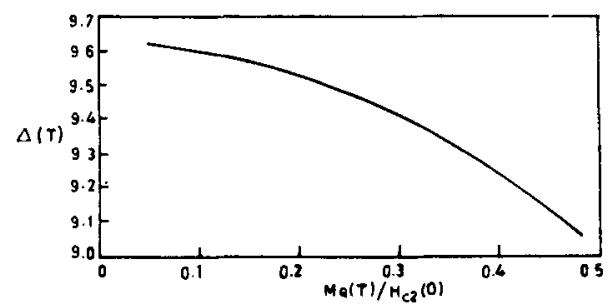

Figure 2 Variation of superconducting gap function $\Delta(T)$ with staggered magnetization $M_{Q}(T)$ at a fixed temperature $T=1.2 \mathrm{~K}$ and $T_{N}=1.31 \mathrm{~K} . \Delta(T)$ is in units of $10^{-4} \mathrm{eV}$.

Table 1. Parameters used in calculation of $\Delta(T)$ and $H_{c 2}(T)$

\begin{tabular}{lccccc}
\hline & & $M_{Q}(0)$ & & $N(\mu)$ & \\
$J_{\text {(ev) }}$ & $\mu_{(e v)}$ & $($ Gauss $)$ & $\rho_{0}$ & $N(0)$ & $\beta h \delta$ \\
\hline $10^{-3}$ & 1 & $10^{2}$ & 13.5 & 0.999 & $10^{-3}$ \\
\hline
\end{tabular}




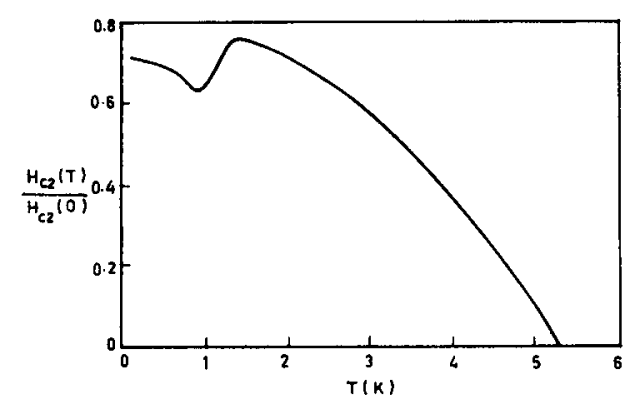

Figure 3. Variation of upper critical field with temperature for $M_{Q}(0) / H_{c 2}(0)=0 \cdot 9$.

of calculation of $\Delta(T)$. The variation of $H_{c 2}(T)$ normalized to $H_{c 2}^{0}(0)$ is plotted in figure 3 for a given value of $M_{Q}(0) / H_{c 2}^{0}(0)=0.9$.

\section{Conclusion}

We note that $\Delta(T)$ and $H_{c 2}(T)$ are modulated owing to the presence of periodic magnetic order in the system. As expected the modulated variation of $\Delta(T)$ with temperature is reflected in the temperature variation of $H_{c 2}(T)$. The curve of $H_{c 2}(T)$ versus $T$ shows the U-shaped depression below $1.31 \mathrm{~K}$ as is observed in experiments.

\section{References}

Das N C 1984 Phys. Rev. B30 2603

Maple M B, Hamaker H C and Woolf L D 1982 in Superconductivity in ternary compounds II (eds) M B Maple and $\Phi$ Fischer (Berlin, Heidelberg, New York: Springer-Verlag) p. 99 and references therein Misra S K and Postma H 1984 Phys. Rev. B30 1285 and references therein

Panigrahi S and Das N C 1989 Philos. Mag. B60 493 